\title{
Mirror reflectometer based on optical cavity decay time
}

\author{
Dana Z. Anderson, Josef C. Frisch, and Carl S. Masser
}

\begin{abstract}
Described is a reflectometer capable of making reflectivity measurements of low-loss highly reflecting mirror coatings and transmission measurements of low-loss antireflection coatings. The technique directly measures the intensity decay time of an optical cavity comprised of low-loss elements. We develop the theoretical framework for the device and discuss in what conditions and to what extent the decay time represents a true measure of mirror reflectivity. Current apparatus provides a decay time resolution of $10 \mathrm{nsec}$ and has demonstrated a cavity total loss resolution of $5 \mathrm{ppm}$.
\end{abstract}

\section{Introduction}

Low-loss optical coatings often play a crucial role in the performance of optical instruments. Unfortunately, coating characteristics can vary considerably from batch to batch from a single manufacturer and drastically from manufacturer to manufacturer, even when identical specifications are quoted for the coating. For this reason it may be useful to have on hand a device which can measure coating characteristics rather than rely on the specifications given by the manufacturer.

In this paper we describe a device designed to measure the reflectivity of highly reflecting mirror coatings (having reflectivity approaching 1), alternatively it can also be configured to measure the transmission of lowloss antireflection coatings (on low-loss substrates). Seemingly straightforward techniques such as measuring the small change in incident light intensity with a power meter have proved unreliable. A technique suggested by Sanders ${ }^{1}$ works well for moderate loss optical elements but less so when losses are very low. The following method is reliable and in fact becomes more accurate with decreasing losses.

The fundamental quantity measured by our apparatus is the cavity decay time. From this quantity, other characteristics such as mirror reflectivity can be inferred. A technique developed by Herbelin et al. ${ }^{2}$ obtains the cavity decay time through a measurement of the phase retardation of an intensity modulated source laser induced by an optical cavity. The technique described herein measures the decay time directly

\footnotetext{
The authors are with California Institute of Technology, Division of Physics, Mathematics, and Astronomy, Pasadena, California 91125.

Received 13 January 1983.

0003-6935/84/081238-08\$02.00/0.

(C) 1984 Optical Society of America.
}

in a straightforward manner using relatively simple instrumentation. Our apparatus has a decay time resolution of $10 \mathrm{nsec}$ and has demonstrated a total cavity loss resolution of $5 \mathrm{ppm}$.

Figure 1 shows a conceptual schematic of the apparatus. Our technique relies on the fact that, with no light incident on the cavity, its output is determined only by its transient response which is characterized by an exponential decay of the intensity with a time constant which in turn is determined only by the round-trip losses in the cavity, the round-trip path length of the cavity, and the speed of light. Therefore if light is injected into the cavity and then the source is quickly shut off, the decay time can be measured with a clock. Measuring the round-trip path length is easy; with these two numbers in hand the cavity losses can be deduced. ${ }^{3}$ To obtain the actual mirror losses one must make a separate measurement of their transmission, which can be done with reasonable precision with a power meter having a large dynamic range (or having calibrated attenuators) by measuring the incident intensity with and without the mirror in the light path. On the other hand, in many applications it may be the actual cavity characteristics which are of interest rather than the mirrors which comprise it, in which case the latter step is unnecessary. Losses of antireflection coatings can be determined by placement in the optical path of a previously characterized cavity. We now delve more deeply into the theory behind the measurement and the calculation of the cavity (and mirror) characteristics and present a small list of convenient formulas relating various essentially equivalent characteristics to the cavity decay time. We subsequently describe the apparatus in more detail and following that we present the results of some tests performed using the apparatus.

\section{Theory}

Imagine an optical cavity which has been filled with light. In a classical sense one may think of the light as 


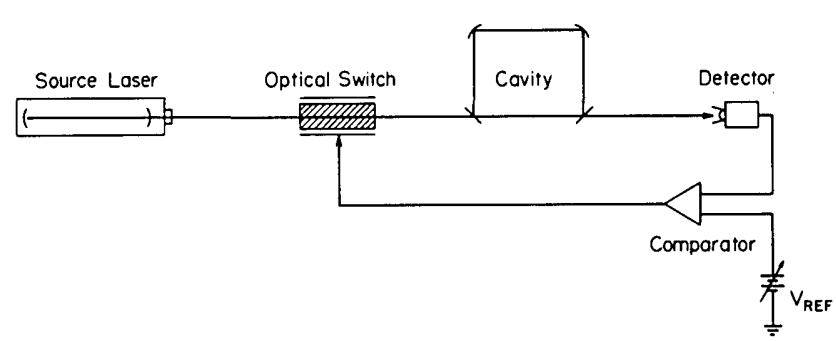

Fig. 1. Conceptual schematic of decay measuring device. Optical switch shuts off light when detector output reaches a preset threshold level.

traveling round and round (or back and forth) along the optical path. With each round trip, a fraction of the light is lost to the outside world. From this simplified picture one might guess that the light at a given point will decay in steps spaced a round-trip time apart with a fractional height dictated by the round-trip losses. With a somewhat greater leap of intuition one might also guess that, if the losses are small, that is, if the step size is small, the decay will be exponential. In the more rigorous treatment below it is shown that this is indeed the case and that in fact the decay time depends only on the round-trip time and the fractional losses. Small fractional changes in cavity length caused, for example, by acoustical vibrations can only make small fractional changes in the decay time. The frequency of the light will affect decay time only insofar as the fractional losses or round-trip time depend on frequency.

As stated above, the transient response of the cavity is exponential only in the case of low total cavity losses. For example, a $10 \%$ total cavity loss implies a standard error of $\sim 1 \%$ for an exponential fit to the cavity decay. Practical considerations, namely, electronic speed, put a more stringent constraint on the maximum permissible loss; so this technique is appropriate only when cavity losses are small. In the following discussions, a low-loss condition is assumed throughout.

There are several ways in which a mirror coating can be characterized, the choice of which often depends on whether the mirror itself or its intended use is to be emphasized. As for the mirror itself, it will suffice for present purposes to ascribe to it three parameters, $R$, $S$, and $T$, the intensity reflection, loss, and transmission coefficients, respectively. We use the term loss and symbol $S$ to represent the nonrecoverable energy losses from scattering and absorption and do not include transmission so that the coefficients are related through energy conservation by

$$
R+S+T=1 \text {. }
$$

We will also use the term total loss meaning the sum, $S$ $+T \equiv \mathbf{L}$ for a given mirror or for the cavity losses, including transmission, as a whole. A mirror may also exhibit amplitude and phase birefringence with respect to the direction of polarization. In general the mirror coefficients will depend on the direction of incidence and wavelength as well; all these dependences will be dropped for the following.
Two or more mirrors may be arranged to form an optical cavity. Suppose light at frequency $\omega$ is incident on one mirror and a detector is placed to monitor the light transmitted through a second mirror as in Fig. 1. To within a phase factor, the electric field at the output is ${ }^{4}$

$E_{0} \exp (-i \omega t)=E_{i} \exp (-i \omega t)\left[C_{m n q}\left(T_{i} T_{o} \mathbf{R}_{p}\right)^{1 / 2} \frac{1}{1-\sqrt{\mathbf{R}} \exp (i \delta)}\right]$,

where $C_{m n q}$ is a mode matching coefficient describing the coupling efficiency between the source and the cavity (typically $m$ and $n$ are small integers, $q$ is a very large integer), $E_{i}$ is the incident electric field, and $T_{i}$ and $T_{o}$ are the transmission of the input and output mirrors, respectively. $\mathbf{R}$ is the product of the mirror reflectivities, $\mathbf{R}_{p}$ is the product of reflectivities of the mirrors which are traversed by the light path in-between (but not including) the input and output mirrors (or $\mathbf{R}_{p}=$ 1 if there are none), and $\delta$ is the round-trip optical phase shift:

$$
\delta=\frac{\omega L}{c}+\varphi_{x}+\varphi_{y},
$$

with $L$ the round-trip path length and $c$ the speed of light. The additional phases $\varphi_{x}, \varphi_{y}$ are dependent on the transverse mode geometry which is in turn dependent on the cavity geometry..$^{5}$ If we focus for the moment on the case where $\delta$ is nearly a multiple of $2 \pi$, that is, near resonance, the factor in brackets in Eq. (2) is seen to have the following character:

$$
\begin{aligned}
H_{m n q}= & C_{m n q}\left(T_{i} T_{o} \mathbf{R}_{p}\right)^{1 / 2}\left\{1-\sqrt{\mathbf{R}}\left[1-i \frac{\Delta \omega L}{c}-\frac{1}{2}\left(\frac{\Delta \omega L}{c}\right)^{2}\right.\right. \\
& \left.\left.+\left(\text { terms higher order in } i \frac{\Delta \omega L}{c}\right)\right]\right\}^{-1},
\end{aligned}
$$

or

$$
\begin{aligned}
H_{m n q} \simeq & C_{m n q}\left(T_{i} T_{o} \mathbf{R}_{p}\right)\left[1-\sqrt{\mathbf{R}}\left(1-i \frac{\Delta \omega L}{c}\right)\right]^{-1} \\
& \equiv A_{m n q} \frac{c}{L}\left[\gamma_{c}+i \Delta \omega_{m n q}\right]^{-1},
\end{aligned}
$$

where

$$
\begin{aligned}
\Delta \omega_{m n q} & =\omega_{m n q}-\omega, \\
\frac{L}{c} \omega_{m n q} & =2 \pi q-m \varphi_{x}-n \varphi_{y}, \\
\gamma_{c} & =(c / L) \frac{1-\sqrt{\mathbf{R}}}{\sqrt{\mathbf{R}}}, \\
A_{m n q} & =C_{m n q}\left[\frac{T_{i} T_{o} \mathbf{R}_{p}}{\mathbf{R}}\right]^{1 / 2} .
\end{aligned}
$$

In some cases it may be desirable to approximate the bracketed factor in Eq. (2) with a sum of $H$ functions [defined by Eq. (4b)] centered about the successive maxima of the bracketed term. This is a reasonable procedure provided the successive maxima are well separated, that is, if the cavity losses are small and if either the transverse mode coupling is small or the transverse mode frequencies are far apart compared to $\gamma_{c}$. 
The field internal to the cavity will become significant only when the source is at or near a resonance of the cavity, that is, when $\delta$ is very nearly a multiple of $2 \pi$. Suppose the cavity has been subject to an electric field on or near its resonance. The magnitude of the field could be varying slowly, but to keep things simple let it remain constant; the result will not change significantly. Now let the source be turned off at $t=0$, quickly but smoothly. What is meant by quickly becomes clear below. In the time domain the source field is

$$
\begin{array}{ll}
E_{i}(t)=E_{s} \exp \left(-i \omega_{s} t\right) & t<0, \\
E_{i}(t)=E_{s} \exp \left[-\left(\gamma_{s}+i \omega_{s}\right) t\right] & t>0 .
\end{array}
$$

To calculate the field at the output we use a Laplace transform approach; the output transform is the product of the input transform and the transfer function of the cavity ${ }^{6}$

$$
E_{o}(s)=E_{i}(s) H(s),
$$

where $s$ is the complex frequency parameter. Equation (6) gives the definition of the transfer function-it is the ratio of output field at frequency $s$ to the input at the same frequency. By inspection of Eqs. (2) and (4), ${ }^{7}$

$$
H(s)=\frac{c}{L} \sum_{m n q} A_{m n q}\left(s+\Omega_{m n q}\right)^{-1},
$$

where the complex frequency $\Omega_{m n q}=\gamma_{c}+i \omega_{m n q}$. For the time being we will concentrate on a single-cavity resonance and replace the subscript triplet with the subscript $c$. The Laplace transform for the input field is

$$
\begin{aligned}
E_{i}(s)= & \int_{0}^{\infty} E_{s} \exp \left(i \omega_{s} t\right) \exp (s t) d t+\int_{0}^{\infty} E_{s} \\
& \times \exp \left[-\left(\gamma_{s}+i \omega_{s}\right) t\right] \exp (-s t) d t \\
= & -E_{s}\left(\Omega_{a}+\Omega_{b}\right)\left(s+\Omega_{a}\right)^{-1}\left(s-\Omega_{b}\right)^{-1},
\end{aligned}
$$

where

$$
\begin{aligned}
& \Omega_{a}=\gamma_{s}+i \omega_{s}, \\
& \Omega_{b}=-i \omega_{s},
\end{aligned}
$$

and the region of convergence for the integration is $-\gamma_{s}$ $<\operatorname{Re}\{s\}<0$. The first integral is derived from times $t$ $>0$, the second from times $t<0$. Equations (7) and (8) yield for the output transform

$$
E_{o}(s)=E_{s} A_{c}\left(\frac{c}{L}\right) \frac{-\left(\Omega_{a}+\Omega_{b}\right)}{\left(s+\Omega_{a}\right)\left(s-\Omega_{b}\right)\left(s+\Omega_{c}\right)} .
$$

Finally, the output field is obtained by taking the inverse transform of Eq. (9). Using the method of residues we find

$$
\begin{aligned}
& E_{o}(t)=E_{s} A_{c} \frac{c / L}{\Omega_{b}+\Omega_{c}} \exp \left(\Omega_{b} t\right) \quad t<0, \\
& E_{o}(t)=E_{s} A_{c}\left[\frac{c / L}{\Omega_{c}-\Omega_{a}} \exp \left(-\Omega_{a} t\right)\right. \\
& \left.+\frac{c / L\left(\Omega_{a}+\Omega_{b}\right)}{\left(\Omega_{a}-\Omega_{c}\right)\left(\Omega_{c}+\Omega_{b}\right)} \exp \left(-\Omega_{c} t\right)\right] \quad t>0 .
\end{aligned}
$$

Substituting for the complex frequencies

$E_{o}(t)=E_{s} A_{c} \frac{c / L}{\gamma_{c}+i \Delta \omega} \exp \left(-i \omega_{s} t\right) \quad t<0$,

$$
\begin{aligned}
& E_{o}(t)= E_{s} A_{c}\left\{\frac{c / L}{\gamma_{c}-\gamma_{s}+i \Delta \omega} \exp \left[-\left(\gamma_{s}+i \omega_{s}\right) t\right]\right. \\
&\left.+\frac{c / L}{\left(1-\gamma_{c} / \gamma_{s}-i \Delta \omega / \gamma_{s}\right)\left(\gamma_{c}+i \Delta \omega\right)} \exp \left[-\left(\gamma_{c}+i \omega_{c}\right) t\right]\right\} \\
& t>0 . \quad(10 \mathrm{~b})
\end{aligned}
$$

Equation (10a) represents the steady-state response of the cavity to the source having been turned on at some early time. Equation (10b) represents the response of the cavity to shutting off the source. ${ }^{8}$ Now what we mean by turning off the source quickly becomes clear: if the source is shut off at a rate $\gamma_{s} \ll \gamma_{c}$, the first term on the right-hand side will dominate although there will be a small amount of interference between the first and second terms for times of the order of the cavity decay time. As expected, the cavity characteristics do not become apparent when the light is slowly extinguished. On the other hand if the light is switched more abruptly so that $\gamma_{s} \gg \gamma_{c}$, the first term in Eq. $10(\mathrm{~b})$ vanishes and the output for $t>0$ becomes

$$
E_{o}(t)=E_{s} A_{c} \frac{c / L}{\gamma_{c}+i \Delta \omega} \exp \left[-\left(\gamma_{c}+i \omega_{c}\right) t\right] .
$$

This last equation says that the electric field at the output decays to zero from its steady-state $(t<0)$ value at a rate characteristic of the cavity. Note too that the frequency of the output field is $\omega_{c}$, the resonance frequency of the cavity-regardless of the original source frequency. The detector at the output senses the intensity of the light transmitted by the cavity. $I(t)=$ $|E(t)|^{2}$. Anticipating the short turn-off time of the input light as in the above discussion, we need only take the square of Eq. (11) to find the characteristic decay time of the intensity:

$$
I(t)=I_{o} \exp \left(-t / \tau_{c}\right),
$$

where

$$
\begin{aligned}
\tau_{c} & =\frac{1}{2 \gamma_{c}}, \\
I_{o} & =\left|E_{o}(t<0)\right|^{2},
\end{aligned}
$$

and the optical frequency time dependence has been omitted. In the above conditions, by measuring the intensity decay time one can obtain the product of the mirror reflectivities since

$$
\frac{\sqrt{\mathbf{R}}}{1-\sqrt{\mathbf{R}}}=2\left(\frac{c}{L}\right) \tau_{c} .
$$

By making a number of measurements with an appropriate number of mirrors one can always obtain the reflectivities of each individual mirror. For example, with three mirrors one can obtain the reflectivity of each by making measurements on each of the three possible two-mirror cavities which can be constructed from them.

Once the cavity decay time and length are known, one can translate these into various other quantities which are often used in optics. For example, one can obtain the finesse of the cavity by inspection of Eq. (12). The finesse is defined as the ratio of the cavity free-spectral 
range (FSR) to the intensity response function full width at half-maximum, $\Delta \nu,{ }^{9}$

$$
\mathbf{F} \equiv \frac{\mathrm{FSR}}{\Delta \nu}=\frac{c}{L}(\Delta \nu)^{-1} .
$$

Keeping terms to second order in $i(\Delta \omega L / c)$ in Eq. (4a),

$$
\begin{aligned}
I(\omega) \propto|H(\omega)|^{2} & =A_{c}^{2}\left(\left\{1-\sqrt{\mathbf{R}}\left[1-\frac{1}{2}\left(\frac{\Delta \omega L}{c}\right)^{2}\right]\right\}^{2}+\mathbf{R}\left(\frac{\Delta \omega L}{c}\right)^{2}\right)^{-1} \\
& =\left(A \frac{c}{L}\right)^{2}\left\{\left[(c / L)(1-\sqrt{\mathbf{R}}) / \mathbf{R}^{1 / 4}\right]^{2}+(\Delta \omega)^{2}\right\}^{-1}
\end{aligned}
$$

thus

$$
\mathbf{F}=\frac{\pi(\mathbf{R})^{1 / 4}}{1-\sqrt{\mathbf{R}}}
$$

In terms of the cavity decay time, using Eq. (13) with $\mathbf{R}$ $\approx 1,10$

$$
\mathbf{F}=2 \pi \frac{c}{L} \tau_{c}
$$

It is sometimes preferable to speak in terms of cavity total loss rather than in terms of mirror reflectivities. When the total losses are small, one can use an easily remembered formula relating the finesse to the cavity losses. Letting $L_{i}=S_{i}+T_{i}\left(=1-R_{i}\right)$ we have, from Eq. (15),

$$
\mathbf{F}=\frac{\pi(\mathbf{R})^{1 / 4}}{1-\sqrt{\mathbf{R}}} \simeq \pi\left\{1-\left[\left(1-L_{1}\right)\left(1-L_{2}\right) \ldots\right]^{1 / 2}\right\}^{-1}
$$

or

$$
\mathbf{F} \simeq \frac{2 \pi}{\sum_{i} L_{i}},
$$

where in the numerator we have set $\mathbf{R}^{1 / 4} \simeq 1$ and where the index $i$ runs over the number of mirrors (and possibly over intracavity element losses as well). In other words the cavity finesse is $2 \pi$ divided by the sum of the losses in the cavity. So far we see that the cavity can be characterized in three equivalent ways: by its decay time, its finesse, and its losses. We can also attribute to the cavity a quality factor defined as its angular resonant frequency times the stored energy divided by the energy loss rate. In terms of the intensity decay time

$$
Q=2 \pi \nu \tau_{c},
$$

where $\nu$ is the optical frequency. ${ }^{11}$

The formulas relating the various cavity parameters in terms of the decay time are summarized in Table I. In the table we have introduced the round-trip number $\kappa=\tau_{c} c / L$. It should be kept in mind that these formulas have been derived in the limit of low total loss.

\section{Accuracy Limitations}

A few comments about the formulas leading to those in Table I are in order. If one were to compare the intensity decay time as given by Eq. (13) with that which is given by the Fourier transform of Eq. (14), one would see a discrepancy of a factor of $\mathbf{R}^{1 / 4}$. This factor was set to 1 in order to get Eq. (16) from Eqs. (13) and (15). The reason for the discrepancy is a bit subtle. The
Table I. Summary of Formulas Relating Various Cavity Parameters to the Cavity Decay Time in the Low-Total Low-Loss Limit ${ }^{\text {a }}$

\begin{tabular}{lcl}
\hline \multicolumn{1}{c}{ Parameter } & Symbol & Formula \\
\hline Cavity linewidth (FWHM intensity) & $\Delta \nu$ & $\left(2 \pi \tau_{c}\right)^{-1}$ \\
Cavity quality factor & $Q$ & $2 \pi \nu \tau_{c}$ \\
Finesse & F & $2 \pi \kappa$ \\
Total cavity loss (including transmission) & L & $\kappa^{-1}$ \\
Mirror reflectivity product & $\mathbf{R}$ & $\left(1-\frac{1}{2 \kappa}\right)^{2}$ \\
$\begin{array}{l}\text { Mirror reflectivity (two-mirror cavity with } \\
\text { identical mirrors) }\end{array}$ & $R$ & $1-\frac{1}{2 \kappa}$
\end{tabular}

${ }^{a} \tau_{c}$ is the cavity intensity decay time, $\nu$ is the optical frequency, $\kappa$ is the round-trip number $\kappa=(c / L) \tau_{c}$, and $L$ is the round-trip optical path length.

output from the cavity is a series of steps having decreasing depths. We are approximating these steps with an exponential. One obtains slightly different values of the decay time depending on whether the exponential is made to fit the electric field decay or made to fit the ir tensity decay. What is this discrepancy trying to te us? The width of the steps at the output is $L / c$, the round-trip travel time. Thus, the best one would expect to measure the decay time is $\pm 1 / 2(L / c)$ regardless of the speed and accuracy of the electronics. The fractional error in a measurement of $\tau_{c}$ is then $1 /$ $(2 \kappa)=L /\left(2 c \tau_{c}\right)=\mathrm{L} / 2$. Any quantity derived from a measured decay time must, of course, reflect this error. For example, a nominal loss of $10^{-2}$ can be measured at best to $\pm 5 \times 10^{-5}$. The error in timing is enough to make any discrepancies in factors of $\mathbf{R}^{1 / 4}$ unresolvable. In fact, it is just large enough to make factors of $\sqrt{\mathbf{R}} \approx$ $1-1 / 2 \mathbf{L}$ unresolvable. The conclusion is that, for the above reasons, it is appropriate to set factors of $\sqrt{\mathbf{R}} \simeq$ 1 as has been done in the table. One can also conclude that a clock resolution beyond the cavity round-trip time is superfluous for a measurement of $\tau_{c}$.

It has been shown that mirror reflectivities can be obtained by measuring the transient response of the cavity and the cavity length, and it is clear from Eq. (10) in what conditions the transient response of the cavity will dominate the output of the cavity. We now present the general design of our apparatus and discuss the requirements on the individual components necessary to meet the above conditions and to have optimum performance.

\section{Apparatus}

The apparatus depicted in Fig. 1 is shown in Fig. 2 with greater detail. Let us iterate the operation of the instrument: light from the source laser impinges on a cavity mirror. The light from the laser will tend to drift in and out of the various cavity resonances. If the laser light is at a resonant frequency of the cavity for a reasonable amount of time, light will build inside the cavity and be transmitted throughout the mirrors of the cavity. A detector senses the intensity of the light transmitted 


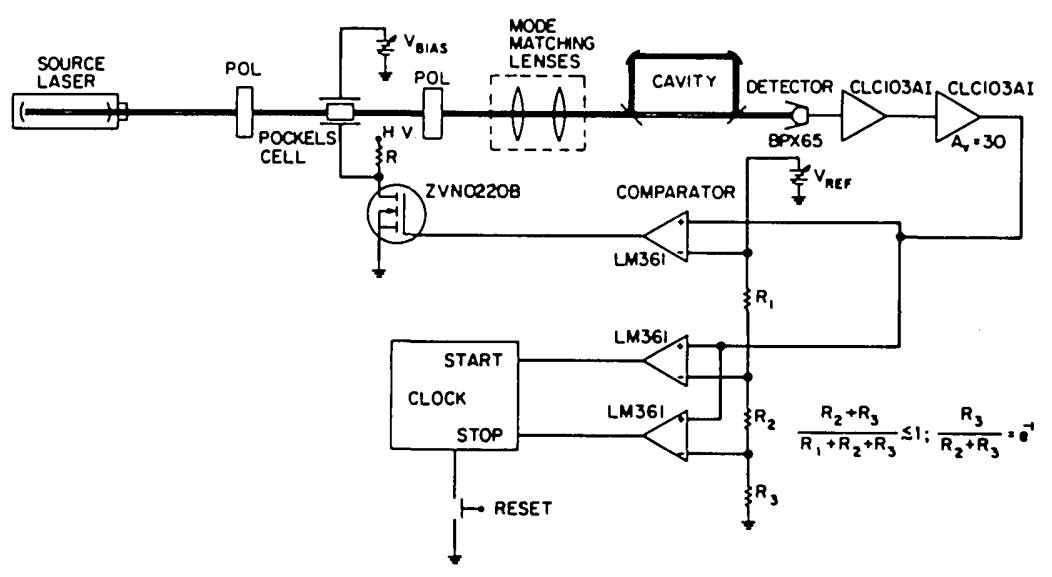

Fig. 2. Schematic of optical and electrical circuit for cavity decay time measurement.

through one of the mirrors; when the transmitted light reaches a predetermined level, the laser light is switched off via the Pockels cell. The detector monitors the decaying light level and a clock measures the 1/e fall time. We now describe the requirements on each of the major components of the system.

The wavelength of the source laser must of course be that for which the mirrors are designed, however, the stability requirements are not severe. Frequency drift is even desirable; in practice we have merely allowed the laser to drift into cavity resonance, thus precluding the need for some means of frequency control or sweeping mechanism.

The mode matching system shown schematically in Fig. 2 is designed so that the laser and cavity fundamental modes are well coupled. ${ }^{4}$ In most cases we have found that the mode matching is not extremely critical; it serves three purposes. In the case of a weak source laser (such as most He-Ne lasers) it is necessary to maximize the output intensity of the cavity and thereby minimize the subsequent requirements on amplifier of gain and signal-to-noise ratio. It may also be necessary to discriminate against off-axis ( $m$ and/or $n \neq 0$ ) cavity modes. In principle, so long as no apertures are present and the mirror surfaces are large, the decay time for all modes is the same. On the other hand, off-axis modes have an effectively larger cross-sectional area and therefore sample a larger surface area on the mirror than do longitudinal $(m, n=0)$ modes. Thus in practice these mode types may have different decay times. Proper mode matching also minimizes the chance of multiple cavity spatial mode excitation. If two or more modes are excited in the cavity, there will be superposition of terms on the right-hand side of Eq. (11), each with a different frequency and possibly a different decay time. However, the eigenmodes with a common mode number $q$ are an orthogonal set. Therefore if the photodetector area is large compared to the spot size, it will detect no interference between modes having the same mode number $q$ even though they may have different frequencies; i.e., the detector sees only the decay of the sum of the intensities of the various modes. Still, if their decay times are different, the net intensity decay can appear nonexponential. In any case, it is probably best if the longitudinal mode coupling is the largest.
The optical switch consists of a Pockels cell (Coherent model 28) sandwiched by a pair of crossed polarizers and some electronics associated with the cell. The bias on the cell is adjusted so that, when the (high-voltage) electronic switch is off, no light is transmitted through the second polarizer. With the switch on, maximum light is transmitted. The second polarizer also determines the state of polarization which will enter the cavity.

The cavity itself can, in general, have any desired geometry; it is best however to. use the highest two transmitting mirrors as input and output if the mirrors of the set are not nominally identical. Motions of the mirrors due, for example, to mechanical vibrations are usually small over time scales of the cavity decay time. Once the light is switched off, motion of the mirrors cannot significantly change the decay time since the fractional change in cavity length is negligible.

The electronics is the most critical part of the apparatus simply because it must be fast. Typical decay times of low-loss cavities may be from 0.1 to $50 \mu \mathrm{sec}$. The precision of the measurement is directly determined by the speed of the electronics.

The front end of the electronics consists of a silicon photodetector (Centronics BPX 65) followed by a cascaded pair of low-noise wideband preamplifiers (Comlinear CL103AI). The output of the amplifier pair is fed to a set of three comparators in parallel. Figure 3 shows the event timing of the comparators. When the output level from the detector reaches a preset thresh-

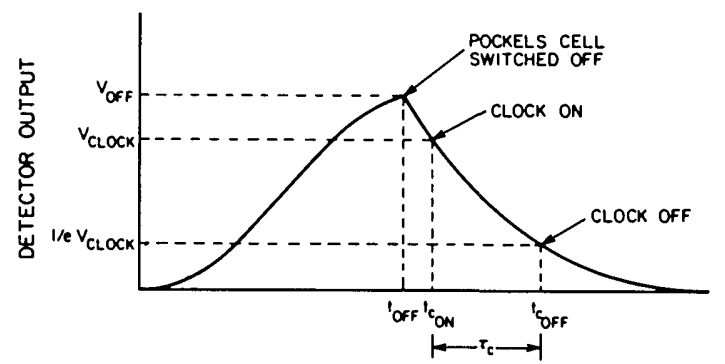

Fig. 3. Comparator timing. Comparator 1 turns the Pockels device off when detector output reaches $V_{\text {off }}$, comparator 2 turns the clock on at $V_{\text {clock}}$, and comparator 3 turns the clock off when the detector output falls to $1 / e V_{\text {clock. }}$ 
old comparator 1 triggers. Its action triggers a highvoltage MOSFET switch which turns the Pockels cell off. Because there is a short delay before the light shuts off, the clock does not begin timing until the detector level falls below a second threshold set somewhat lower than the first. The threshold on the third comparator is $1 / e$ of the second threshold. When this comparator fires, the clock ceases timing. The decay time is displayed directly. Once this time is recorded the cycle may be restarted by resetting the clock and turning on the Pockels cell.

It is best to adjust the threshold of the first comparator so that only events with the highest intensity trigger the cycle. One can do this by first setting the threshold high, then slowly reducing it until the circuit triggers. One should then have a good working threshold level. Multimode events caused by fast laser drift or large and fast cavity mirror motions tend to be less intense since the laser light is spending relatively less time on resonance than when drift and motions are slow and small. By setting the threshold high one avoids most undesirable (nonexponential) events.

In our circuit the comparators are specified as having a 14-20-nsec propagation delay. If overall timing accuracy needs to be much better than $10 \mathrm{nsec}$, it would be wise to select a matched pair for the second and third comparators which control the clock. Propagation effects can be minimized this way.

Our clock consists of a set of TTL counters driven by a quartz crystal and interfaced to a digital display. An alternative would be a commercial unit such as a Fluke $7261 \mathrm{~A}$ counter which has a 10-nsec resolution and can be used in an interval timer mode. Another alternative is a fast storage oscilloscope which has the additional advantage of making the second and third comparator unnecessary; this however is an expensive choice.

The Pockels cell driver is a high-speed high-voltage MOSFET switch. This switch must of course be capable of switching a high voltage $(\sim 200 \mathrm{~V})$ in a time rather short compared with the cavity decay time.

\section{Tests}

For the most part there is a good deal of freedom in the design of the instrument and requirements on the optical components are rather lax. It is the electronics which bears the brunt of the constraints. Before measuring actual mirror characteristics we performed several tests of the decay time device to confirm that it was indeed measuring cavity losses and doing so accurately. Our tests were performed using essentially the arrangement shown in Fig. 2 except that a two-mirror cavity was used rather than a ring cavity. The source is a single-frequency argon-ion laser at $514.5 \mathrm{~nm}$. In each test the mode matching lenses were adjusted to obtain reasonable coupling efficiency into the cavity and yet not so much care was taken to avoid coupling to off-axis modes.

The overall speed of the electronics was tested by placing the detector directly in front of the source and attenuating the source intensity to simulate light from the cavity. Since light is impinging directly onto the detector the Pockels cell is immediately switched off on activation of the comparator circuitry. The decay time was measured to be $\sim 25 \mathrm{nsec}$. This fall time is dominated by the turn-off time of the Pockels cell; it does not, however, inflict a serious limitation to the precision of the decay time measurement for nominal decay time of $0.1 \mu \mathrm{sec}$ or more. The trigger circuitry is designed to circumvent the problems of incident light intensity fall time.

The mirrors that we wanted to characterize were designed for use in our 40-m interferometer. For this reason we chose to measure these mirrors using a $10-\mathrm{m}$ test facility. The resulting beam spot size samples a relatively large fraction of mirror surface. The cavity optical path was evacuated: we have made tests in open air cavities but found our results depended on the ambient Pasadena air quality.

The detector was replaced behind the cavity in order to make a measurement of the cavity decay time. A Tektronics digital storage oscilloscope (model 468) was used to monitor the cavity decay. A storage oscilloscope is a very useful diagnostic tool in this application. It can be used to verify the exponential nature of the decay, but we have also found it superfluous as long as the maximum-threshold rule of thumb is followed.

To verify the calibration of the instrument and to demonstrate that the cavity decay is indeed exponential, we first measured the decay time of the cavity. We then chose a resistor and capacitor pair having a $\mathrm{RC}$ constant equal to the measured decay time. To well within the $1 \%$ uncertainty in the $\mathrm{RC}$ component values, the two time constants agreed. Figure 4, recorded with the digital storage oscilloscope, illustrates the results. The cavity decay curve is the lower of the two curves on the left-hand side of the photo. The RC event is the upper curve. The actual decays are perfectly merged within

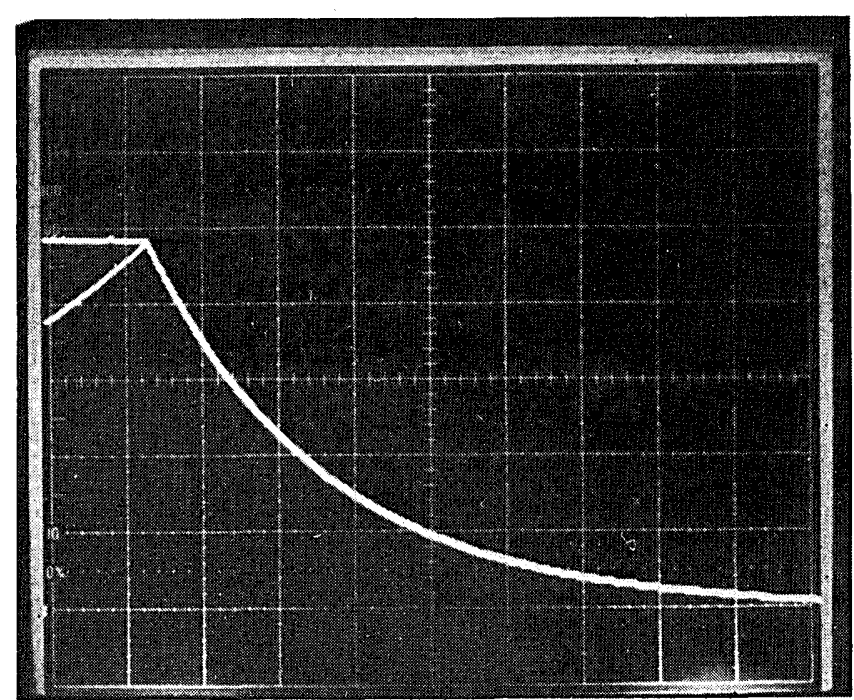

Fig. 4. Typical cavity decay curve from a two-mirrored 10-m cavity having a fall time of $23.2 \mu \mathrm{sec}$ starts on the left-hand side as the lower of the two curves. The second curve is the decay from a $\mathrm{RC}$ network having the same decay constant. The decays from the two events are perfectly merged showing that the cavity decay is indeed exponential. 


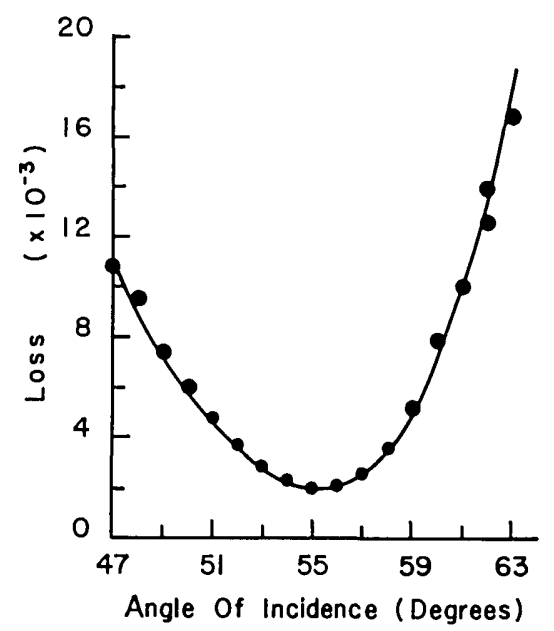

Fig. 5. Cavity loss vs tilt of an etalon near the Brewster angle. Dots are experimental data. The theoretical (solid line) curve was obtained assuming a nominal cavity loss of $2 \times 10^{-3}$ and a Brewster angle of $55.25^{\circ}$.

the digitalizing accuracy of the oscilloscope. The cavity decay is thus seen to be exponential. Direct measurement of the threshold voltages for clock turn-on and turn-off give a calibration for $e^{-1}$ fall time of $0.5 \%$.

As a third check of the device we measured the decay time of our $40-\mathrm{m}$ interferometer which currently uses rather low reflectivity mirrors. The intensity linewidth of this cavity had been previously measured to be $6.0 \pm$ $0.5 \mathrm{kHz}$ by scanning the frequency of a stabilized laser and monitoring the intensity of the transmitted light. The decay time deduced from the linewidth is $27 \pm 2$ $\mu$ sec. This is in good agreement with the $26.5 \mu \mathrm{sec}$ measured directly. This test shows that the decay time measurement agrees with an independent measure of cavity loss.

As a final test of the instrument, we measured the loss of an uncoated etalon placed in an optical cavity near the Brewster angle. For simplicity, this was done using an open air cavity rather than an evacuated cavity (71-cm spacing between mirrors). The cavity loss was calculated from the measured decay time for small angles about the Brewster angle. Figure 5 shows the theoretical fit (solid line) to the data (dots) assuming a nominal cavity loss of $2 \times 10^{-3}$ and a Brewster angle of $55.25^{\circ} .12$ The loss shown counts two surfaces. The size of the dots indicates approximately one standard error. The fit to the data is very good, indicating that the decay time device is indeed measuring the actual loss of the inserted optical element.

We intended to employ this device to characterize mirrors of unknown reflectivity. We now demonstrate the use of this instrument for such a purpose.

The $10-\mathrm{m}$ cavity decay time was measured using two different cavities in vacuum: one having low total loss, the other comparatively high loss. Both members of each pair of mirrors came from a single-coating run and so were considered identical. In both cases, the cavities consisted of one flat mirror and one curved mirror with a radius of $62 \mathrm{~m}$.
The decay time for the low-loss cavity was $185 \mu \mathrm{sec}$ $\pm 1.4 \%$ where the error is the rms deviation from the mean of twenty consecutive measurements. Transmissions of these mirrors was measured to be $120 \mathrm{ppm}$. The decay time implies a $180-\mathrm{ppm}$ total loss per mirror, consistent with the transmission measurement and allowing for $60-\mathrm{ppm}$ absorption and scattering loss. The second cavity decayed in $26.24 \mu \mathrm{sec} \pm 0.2 \%$, indicating a total loss per mirror of $1270 \mathrm{ppm}$. These mirrors are evidently rather lossy as their transmission was measured to be $622 \mathrm{ppm}$. Note that in both cases the standard error implies a total loss per mirror resolution of $2.5 \mathrm{ppm}$ (although in the second case the $0.5 \%$ timing calibration exceeds the $0.2 \%$ spread in decay times). For the record, our measurements give $\mathbf{R}=0.999820 \pm$ $3 \mathrm{ppm}$ and $\mathbf{R}=0.998730 \pm 7 \mathrm{ppm}$ for the low- and high-loss mirrors, respectively.

\section{Conclusions}

We have presented the theoretical foundations behind the decay time measurement and have discussed in what conditions a measurement of the decay time represents a measure of the mirror characteristics. We have furthermore discussed problems which can be encountered in a measurement. With the decay time and cavity length in hand one can choose to characterize either the cavity or the mirrors in a variety of ways using the formulas of Table I.

The decay time apparatus was designed with a resolution of 10 nsec and has exhibited a total loss resolution of $5 \mathrm{ppm}$. This was accomplished by merely measuring the time interval between two voltage levels on the decay curve. One can envision various improvements such as monitoring an entire decay and fitting to it an exponential curve or improving the clock resolution to better than 10 nsec. We expect the technique could be pushed; nevertheless, this straightforward approach shows very respectable performance using rather simple instrumentation and measurement procedures.

We owe special thanks to R. W. P. Drever for his encouragement and insight. We would also like to thank R. E. Spero, S. E. Whitcomb, and M. Hereld for valuable advice and assitance. We are indebted to T. Hutchings and S. W. Hammons of Litton Industries, Guidance \& Control Systems Division, for providing us with the low-loss (180-ppm) mirror coatings and to S. S. M. Lu and R. Hargrove of Litton for the production of the coatings.

The work of D. Z. Anderson was supported by NSF grant PHY82 04056.

\section{References}

1. V. E. Sanders, Appl. Opt. 16, 19 (1977); V. E. Sanders, Rockwell International; private communication.

2. J. M. Herbelin, J. A. McKay, M. A. Kwok, R. H. Ueunten, D. S. Urevig, D. J. Spencer, and D. J. Benard, Appl. Opt. 19, 144 (1980). 
3. The measurement of decay time to determine cavity losses is by no means original to us. The technique has its roots in passive RLC circuit theory and in microwave networks. Although cavity decay time is discussed in the literature (compare Ref. 9), we are not aware of any published work describing apparatus or experiments making use of it. We are, however, aware that techniques measuring cavity decay have indeed been used by some individuals to measure optical cavity characteristics. A rotating mirror has been used to gate laser light into an optical cavity (M. Ford, Ph.D. Thesis, U. Glasgow, Scotland, 1979, unpublished). Madey uses a similar technique to measure the decay time of a freeelectron laser cavity (J. Madey, Stanford U.; private communication).

4. M. Born and E. Wolf, Principles of Modern Optics (Pergamon, New York, 1980). Equation (2) of the text is a generalization of Eq. (12), p. 325, in Born and Wolf.

5. Compare H. W. Kogelnik and T. Li, Appl. Opt. 5, 1550 (1966); S. A. Collins, Jr., Appl. Opt. 3, 1263 (1964).

6. We have not rigorously justified the use of the Laplace transform method for optical cavities. Replacement of the laser and cavity by equivalent electrical circuits lends credence to our procedure. Such equivalent circuits have been studied, for example, by $\mathrm{E}$. I. Gordon, Bell Syst. Tech. J. 43, 507 (1963).

7. On Laplace transform calculus see, for example, B. P. Lathi, Signals Systems and Communication (Wiley, New York, 1965).

8. The scrutinizing reader will have noted that the boundary condition that $E\left(t=0^{-}\right)=E\left(t=0^{+}\right)$is not met in Eq. (10) because of the omission of the phase factor in Eq. (2). The condition can be regained by replacing $t$ on the right-hand sides of Eqs. (10) by $t-t_{0}$ where $t_{0}$ is the light travel time from the input to the output mirror.

9. Ref. 4 , p. 328 .

10. We have derived formulas assuming pointlike losses. The problem of distributed losses is discussed by A. Yariv, Quantum Electronics (Wiley, New York, 1975), p. 141.

11. A. Yariv, Introduction to Optical Electronics (Holt, Rinehart, \& Winston, New York, 1971), p. 79.

12. Ref. 4, p. 42.

Books continued from page 1225

Laser Spectroscopy, Vol. 6. Edited by H. P. WEBER and W. LUTHY. Springer-Verlag, Heidelberg, 1983. 442 pp. $\$ 29.00$.

Lasers continue to be a very valuable tool for spectroscopic investigations. In particular, many studies involving high resolution, sensitivity, or unstable atoms and molecules that were difficult or not possible with conventional spectroscopy can now be done. The latest of these investigations is reported in this book, which contains the proceedings of the Sixth International Conference on Laser Spectroscopy, Interlaken, Switzerland, 27 June-1 July 1983. (Previous meetings started in 1973 and have been held every other year since then. Publications of the proceedings of these conferences are also available from Springer-Verlag.)

A total of 145 papers are divided into 16 sections: (1) Photons in Spectroscopy; (2) Spectroscopy of Elementary Systems; (3) Coherent Processes; (4) Novel Spectroscopy; (5) High Selectivity Spectroscopy; (6) High Resolution Spectroscopy; (7) Cooling and Trapping; (8) Collisions and Thermal Effects on Spectroscopy; (9) Atomic Spectroscopy; (10) Rydberg-State Spectroscopy; (11) Molecular Spectroscopy; (12) Transient Spectroscopy; (13) Surface Spectroscopy; (14) NL-Spectroscopy; (15) Raman and CARS; and (16) Double Resonance and Multiphoton Processes.

All the papers are short (1-6 pages) concise statements of the research reported, and most contain extensive references and excellent' figures. To understand these papers one must be acquainted with the fundamentals of the apparatus and the phenomena being investigated. They are written for the specialist in this work. This book is a welcome addition to the literature on spectroscopy but in no way should it be confused with a text on laser spectroscopy.

The papers in this book, like the papers in this journal, are valuable references for all people who desire to keep up with the rapidly changing field of laser spectroscopy.

DAVID W. STEINHAUS

Molecular Light Scattering and Optical Activity. By L. D. BARRON. Cambridge University Press, New York, 1983. 408 pp. $\$ 69.50$.

This volume provides a comprehensive introduction to the molecular theory of optical activity phenomena. The author has been one of the main contributors to this field for the past 15 years; he is especially well known for his work on Rayleigh and Raman optical activity.

Optical activity originates in a difference in response of a molecular system to right and left circularly polarized light. The first chapter provides a review of optical activity phenomena including brief histories of their discovery and short and clear descriptions of the essential physics of each of them. The author uses the term "natural" to denote optical activity phenomena in the absence of externally imposed electric and magnetic fields. He discusses natural and magnetic optical rotation and circular dichroism, light scattering from optically active molecules, vibrational optical activity, the Kerr and Cotton-Mouton effects, as well as some aspects of symmetry in relation to optical activity.

The second chapter contains an introduction to the theory of atoms and molecules in electric and magnetic fields. The theoretical description uses Maxwell's equations as a starting point. The Stokes parameter representation is used to represent the polarization properties of light waves, and a full discussion of partially polarized light is given. Quantum mechanical perturbation theory is invoked to derive expressions for the molecular property tensors that characterize the responses of a molecule to particular electric and magnetic field components. This chapter will be of special interest to Raman spectroscopists for its clear discussion of Raman transition polarizabilities.

The third chapter as the author says "constitutes the heart of the book." It uses the theoretical material derived in the previous chapter to calculate explicit expressions, in terms of molecular property tensors, for the polarization and intensity of light scattered by a molecular system. These expressions form the basis of all the theories of optical activity phenomena presented in the rest of the book. The author gives a clear review of the relationship between scattering and refraction and presents detailed expressions for polarization effects in both scattering and refraction.

Chapter 4 as the author states is a "... rambling affair. It collects together a number of disparate theories all of which have some bearing on the application of symmetry arguments to molecular properties in general and optical activity in particular." This chapter includes discussions of Cartesian tensor algebra, inversion symmetry, and permutation symmetry.

The last four chapters of the book contain detailed theories of particular optical activity phenomena, usually including discussions of the molecular structural features that give rise to them. The most well-established optical activity phenomena in chemistry-natural and magnetic electronic optical rotation and circular dichroism-are discussed in Chaps. 5 and 6. In Chaps. 7 and 8 the emphasis changes to the newer topics of natural vibrational optical activity, antisymmetric scattering, and magnetic Raman optical activity. 